\title{
Airborne pathogen projection during ophthalmic examination
}

\author{
Basak Bostanci Ceran ${ }^{1}$ (D) Alp Karakoç ${ }^{2}$. Ertuğrul Taciroğlu ${ }^{3}$
}

Received: 6 May 2020 / Revised: 17 June 2020 / Accepted: 19 June 2020 / Published online: 25 June 2020

(C) Springer-Verlag GmbH Germany, part of Springer Nature 2020

\begin{abstract}
Purpose Microscale droplets act as coronaviruses $(\mathrm{CoV})$ carriers in the air when released from an infected person and may infect others during close contact such as ophthalmic examination. The main objective of the present work is to demonstrate how CoV deposited droplets are projected during biomicroscopy and to discuss what kind of precautions should be taken in ophthalmic practice.

Methods A coupled fluid-structure system comprising smoothed particle hydrodynamics and the finite element method has been built to assess the projection of droplets spreading from an infected person. Different conditions based on the maximum exit flow velocity from the infector's mouth during the ophthalmic examination were modeled.

Results During exhalation, for which the exit flow is $\sim 1000 \mathrm{~mm} / \mathrm{s}$, the average horizontal distance of the flow front was $200 \mathrm{~mm}$ while individual particles can reach up to $\sim 500 \mathrm{~mm}$. In case of coughing or sneezing (corresponding to an exit flow of $\sim$ $12,000 \mathrm{~mm} / \mathrm{s}$ ), the average horizontal distance of the flow front was $\sim 1300 \mathrm{~mm}$.

Conclusion During the ophthalmic examination, the proximity to the patient's nose and mouth was observed to be less than the horizontal distance of flow front particles. Even though mounted breath shields are used, particles flew beyond the shield and contaminate the ophthalmologist. Compared with the current protective breath shields, the use of a larger shield with a minimum radius of $18 \mathrm{~cm}$ is needed to decrease viral transmission.
\end{abstract}

Keywords Airborne pathogens · Biomicroscope $\cdot$ Coronavirus · COVID-19 · Droplet projection · Fluid-structure system · Ophthalmology $\cdot$ SARS-CoV-2 $\cdot$ Viral transmission

\section{Introduction}

In December 2019, China reported a pneumonia outbreak in Wuhan, a city with more than 11 million people [1]. The causative organism was identified as a new coronavirus-

This article is part of the Topical Collection on Perspectives on COVID-19

Electronic supplementary material The online version of this article (https://doi.org/10.1007/s00417-020-04815-4) contains supplementary material, which is available to authorized users.

Basak Bostanci Ceran drbbostanci@gmail.com

1 Faculty of Medicine, Ophthalmology Department, Okan University, İstanbul, Turkey

2 Department of Bioproducts and Biotechnology, Aalto University, Espoo, Finland

3 Civil and Environmental Engineering, University of California, Los Angeles, CA, USA namely, novel coronavirus: nCOV-severe acute respiratory syndrome coronavirus 2 (SARS-CoV-2) - and the disease as the coronavirus disease 2019 (COVID-19) [2]. After its introduction, the World Health Organization declared the situation as a public health emergency of international concern [3] and published suggestions for protection and prevention of transmission [4].

Respiratory droplets, aerosols, and direct contact are confirmed transmission routes for COVID-19 infection [5, 6]. Droplets and aerosols might carry the virus in the air when they are spread from an infected person by breathing, sneezing, or coughing [7]. Individuals who were infected by subclinical patients by droplets or by contact with secretions have also been reported [8]. In addition to that, some anecdotal reports suggest the possibility of transmission by aerosols through the conjunctival route if no eye protections are used $[9,10]$.

The diameter of the droplets spreading from an infected individual through breathing, coughing, and sneezing may range from 0.5 to $200 \mu \mathrm{m}$ and transmission may be possible over a short distance (up to $90 \mathrm{~cm}$ ) in the air [11]. Droplets 
which are smaller than $100 \mu \mathrm{m}$ have a high potential to be accumulated in the pharynx and larynx of the infected person and cause further infection [12].

Ophthalmologists work in close proximity with patients during slit-lamp examinations (Fig. 1a). Although protective measures like wearing masks, goggles, and breath shields (Fig. 1b) are taken in large epidemics like COVID-19, in daily practice, biomicroscopes are typically used without any prevention. In consideration of these occasions, the present study aims to simulate airborne pathogen projection through breathing, coughing, and sneezing during the ophthalmic examination and suggest preventive measures for diminishing transmission.

\section{Methods}

\section{Numerical methods: smoothed particle hydrodynamics}

In the present study, smoothed particle hydrodynamics (SPH), which is a mesh-free Lagrangian particle method that allows functions to be expressed in terms of their values at a set of disordered particles [13], was implemented to study the airborne pathogens spreading under different circumstances. SPH is a versatile and stabile method that address modeling needs where traditional numerical models such as the finite element method (FEM) and finite difference method (FDM) are inefficient [14]. Especially for the interactive applications for the highly deformable bodies and fluid flows, SPH is proven to be a convenient method, which has been validated with various experiments and benchmark problems in the literature [15-18]. In the SPH framework, continuous field quantities $A$, their gradients $\nabla A$, and Laplacian $\nabla^{2} A$ at $i^{\text {th }}$ particle position $\boldsymbol{x}_{i}$ are interpolated as a weighted sum of contributions from the neighboring particles as

$$
\begin{aligned}
& A\left(x_{i}\right)=\sum_{j} m_{j} \frac{A_{i}}{\rho_{j}} W\left(x_{i}-x_{j}, h\right), \\
& \nabla A\left(x_{i}\right)=\sum_{j} m_{j} \frac{A_{j}}{\rho_{j}} \nabla W\left(x_{i}-x_{j}, h\right), \\
& \nabla^{2} A\left(x_{i}\right)=\sum_{j} m_{j} \frac{A_{j}}{\rho_{j}} \nabla^{2} W\left(x_{i}-x_{j}, h\right),
\end{aligned}
$$

Here, $j$ is the particle index, $m$ and $\rho$ are the particle mass and density, respectively, $h$ is the radius of support (or smoothing length bounding the neighboring particles used in the calculations), and $W\left(\boldsymbol{x}-\boldsymbol{x}_{j}, h\right)$ is the smoothing kernel that is chosen as a quintic function [19].

$$
\begin{aligned}
& W\left(x_{i}-x_{j}, h\right)=\frac{3}{359} \pi h^{3} \cdot w\left(\frac{\left\|x_{i}-x_{j}\right\|}{h}\right), \\
& w(q)=\left\{\begin{array}{c}
(3-q)^{5}-6(2-q)^{5}+15(1-q)^{5} ; 0 \leq q<1 \\
(3-q)^{5}-6(2-q)^{5} ; 1 \leq q<2 \\
(3-q)^{5} ; 2 \leq q \leq 3 \\
0 ; q>3
\end{array}\right.
\end{aligned}
$$

Since SPH is a Lagrangian-based method, particles move with the domain. Thus, the number of particles, each of which has constant masses $m$, is kept constant during the computations. This inherently satisfies the conservation of mass, where the conservation of momentum in terms of Navier-Stoke's formulation is expressed as

$\rho \frac{D v}{D t}=-\nabla \rho+\mu \nabla^{2} v+\rho g$

where $D v / D t$ is the substantial derivative, $\boldsymbol{v}$ is the velocity, $\boldsymbol{g}$ is the gravitational acceleration, and $\mu$ is the viscosity of the fluid. In the SPH framework, for the $i$ th particle, the pressure and viscous terms on the right-hand side of Eq. (6) are expressed by means of the Eqs. (1)-(3) as [20].
Fig. 1 a A standard ophthalmic examination with the biomicroscope. b Biomicroscope with a protective breath shield
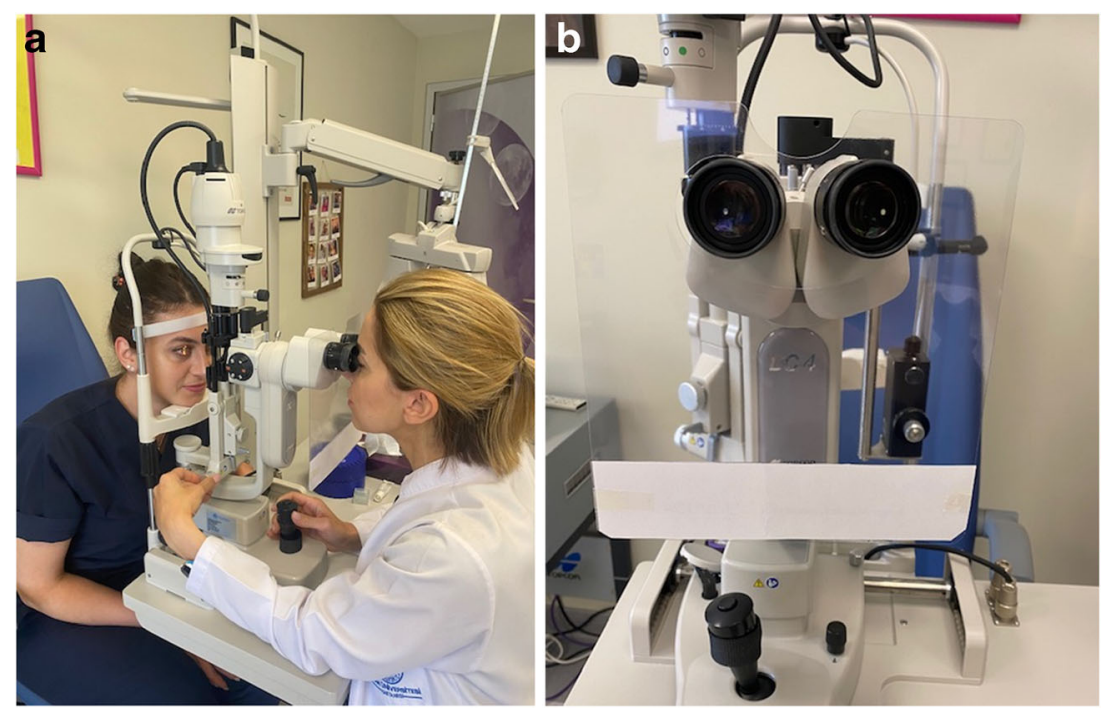
Table 1 Characteristics of the saliva (water) and air

\begin{tabular}{lll}
\hline & Saliva & Air \\
\hline Reference density, $\rho_{0}$ (tonne $\left.\mathrm{mm}^{-3}\right)$ & $1 . \times 10^{-9}$ & $1.225 \times 10^{-12}$ \\
Viscosity, $\mu(\mathrm{MPa} \mathrm{s})$ & $1 . \times 10^{-9}$ & $1 . \times 10^{-11}$ \\
Speed of sound, $c_{\mathrm{s}}\left(\mathrm{mm} \mathrm{s}^{-1}\right)$ & $1.481 \times 10^{6}$ & $3.43 \times 10^{5}$ \\
Material constant $\Gamma_{0}(-)$ & 0. & - \\
Material constant $s(-)$ & 0. & - \\
Gas Constant, $R\left(\mathrm{~mJ}\right.$ tonne $\left.{ }^{-1} \mathrm{~K}^{-1}\right)$ & - & $287.058 \times 10^{6}$ \\
Ambient Pressure, $p_{a}(\mathrm{MPa})$ & - & 0.101325 \\
\hline
\end{tabular}

$-\nabla p_{i}=-\rho_{i} \sum_{j \neq i}\left(\frac{p_{i}}{\rho_{i}^{2}}+\frac{p_{j}}{\rho_{j}^{2}}\right) m_{j} \nabla W\left(x_{i}-x_{j}, h\right)$,

$\mu \nabla^{2} v_{i}=\mu \sum_{i \neq i}\left(v_{i}-v_{j}\right) \frac{m_{j}}{\rho_{j}} \nabla^{2} W\left(x_{i}-x_{j}, h\right)$.

For the stable clusters of particles, the velocity is modified with XSPH (X means unknown) introduced by Monaghan [21], which follows

$v_{i}=v_{i}+\varepsilon \sum_{j} \frac{m_{j}}{\rho_{i}+\rho_{j}}\left(v_{i}-v_{j}\right) W\left(x_{i}-x_{j}, h\right)$,

where $\varepsilon \in[0,1]$.

In this framework, the relationship between the pressure and density for saliva, which is assumed to possess the mechanical characteristics of water, can be expressed with the linear Us-Up Hugoniot form of the Mie-Grüneisen equation of state,

$p=\frac{\rho_{0} c_{s}^{2} \eta}{(1-s \eta)^{2}}\left(1-\frac{\Gamma_{0} \eta}{2}\right)+\Gamma_{0} \rho_{0} E_{m}$.

Here, $c_{s}$ is the speed of sound, $\Gamma_{0}$ and $s$ are material constants, $\rho_{0}$ is the reference density, $E_{m}$ is the internal energy per unit mass, and $\eta$ is the nominal volumetric strain [22].
On the other hand, the air is assumed to be ideal gas with the equation of state given as follows

$p+p_{a}=\rho R\left(T-T_{\text {zero }}\right)$,

where $p_{a}$ is the ambient pressure, $R$ is the gas constant, $T$ is the temperature of the gas, and $T_{\text {zero }}$ is the temperature of the absolute zero [14].

Based on the abovementioned expressions, $D v / D t$ of Eq. (6) is solved in the scheme of explicit central-difference time integration algorithm with Abaqus/Explicit to obtain the time histories of field variables for all the particles. In the simulations, Abaqus built-in surface behavior formulation is used to prevent the particles on opposite sides of a surface from interacting with each other, and free-slip condition is used throughout the mouth structure in consideration to the low viscosity and high flow velocity [14].

\section{Transmission routes during the biomicroscopic examination}

Transmission of airborne pathogens eventuates by aerosol and droplets [23, 24]. Airborne transmission of aerosols $(\leq 5 \mu \mathrm{m})$ may occur over distances greater than $1 \mathrm{~m}$, whereas droplet transmission is the spread of droplets $(>5 \mu \mathrm{m})$ over shorter distances [24]. In the present study, the transmission of all airborne pathogens, both aerosol and droplet, is included because the distance between two individuals during biomicroscopic examination is less than $50 \mathrm{~cm}$.

Droplets and aerosols projecting from the patient's mouth and nose spread to air and contaminate the recipient. Droplet size distribution does not differ between acts with expulsive pressure, such as coughing-sneezing and normal exhalation; however, the number of respiratory droplets may differ $[25,26]$. The pathogen-loaded droplets that are inhaled may than be deposited in the recipient's respiratory tract, although there are reports showing the possibility of mucous membranes to be contaminated [27]. Following this deposition, the pathogen gains the ability to be amplified in the respiratory tract and peripheral tissues of the recipient and the recipient become an infector [28].
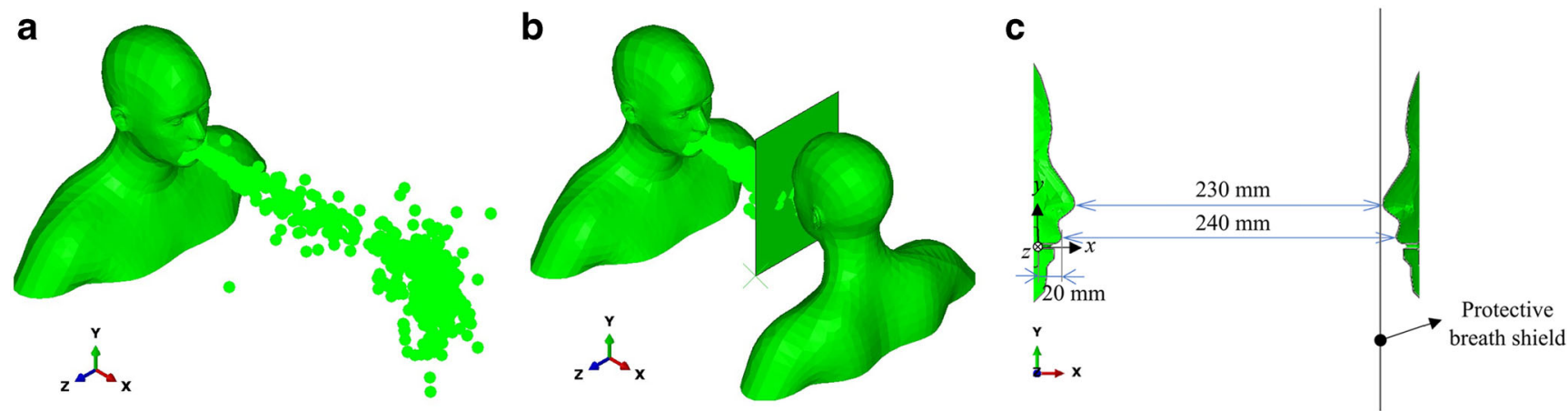

Fig. 2 Configurations: a manikin without any prevention action, $\mathbf{b}$ manikins with protective breath shield, $\mathbf{c}$ representation of the origin in local $x y z$ Cartesian coordinate system and dimensions used for the manikins for the ophthalmic examination simulation 
a
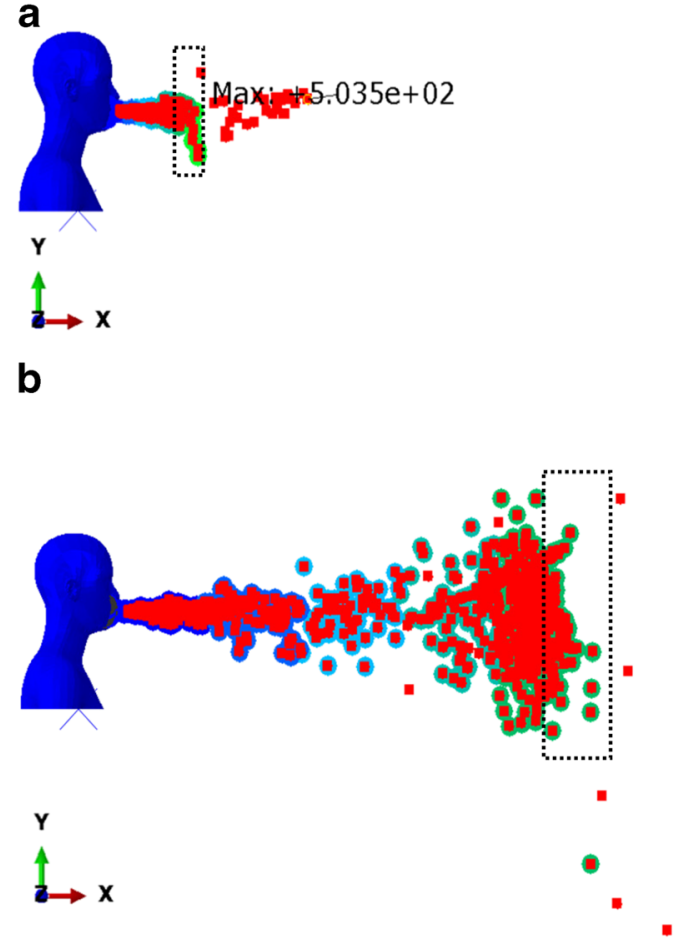

Fig. 3 Airborne particle projection without any precaution, i.e., use of no tissue, mask, or sleeve to cover the face: a exhalation (the maximum flow velocity at the exit from the infector's mouth is $\sim 1000 \mathrm{~mm} / \mathrm{s}$ ), b

\section{Results}

The outflow from the mouth or nose during breathing, coughing, or sneezing can be treated as jet flow with several meters per second [7]. Since each human has a unique mouth structure, way of breathing, coughing, or sneezing mechanisms, the models can only provide a likelihood for the pathogen spreading mechanisms. The characteristics of the saliva (e.g., density, viscosity) can simply vary for each human under different seasonal and ambient conditions. In order to standardize these effects, the flow characteristics were adapted from the experimental investigations in the literature, which have been carried out for various indoor scenarios [29-32]. Based on the indoor investigations, the drag force of the ambient environment was assumed to be negligible. Moreover, due to the short period of analyses, which is $t=1.0 \mathrm{~s}$, evaporation physics was also neglected, whereas gravity was taken into. Accordingly, the maximum exit flow velocity from the infector's mouth, which was modeled as an ellipse with semiminor axis length of $2 \mathrm{~mm}$ and semi-major axis length of $9 \mathrm{~mm}$, was regulated as $\sim 1000 \mathrm{~mm} / \mathrm{s}$ (exhalation) and $12,000 \mathrm{~mm} / \mathrm{s}$ (coughing/sneezing). The solution domain was generated based on the oral volume capacity studies by Nascimento et al., and which composed of a $\sim 70 \mathrm{ml}$ reservoir of air ( $\sim 90 \%$ volume fraction) and saliva particle ( $\sim 10 \%$ volume fraction) mixture, where the air was assumed an ideal gas and saliva inherited the mechanical characteristics of water

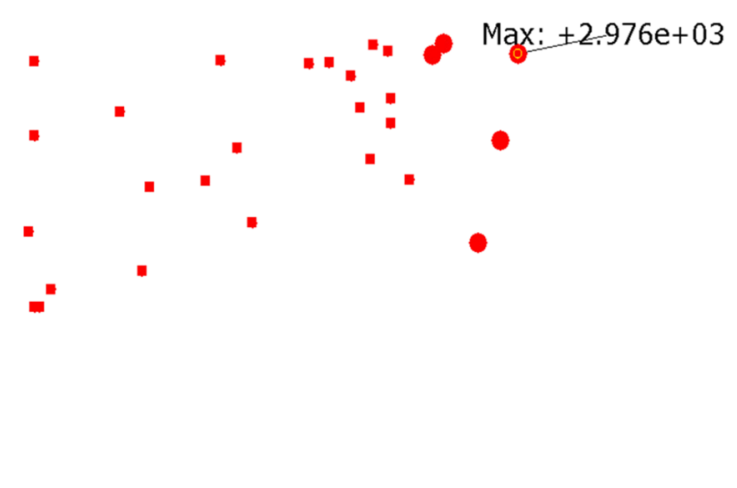

coughing/sneezing (the maximum flow velocity at the exit from the infector's mouth is $\sim 12,000 \mathrm{~mm} / \mathrm{s})$. The dashed lines represent the flow front where the particles are densely packed

[33] as listed in Table 1. The particles were assumed to be spherical with radii of $50 \mu \mathrm{m}$ in accordance with the previous parametric studies [11, 12, 34].

In order to understand the social distancing phenomenon and airborne pathogen spread during the ophthalmic examination, two scenarios with two different conditions based on exit flow velocities were considered:

(a.1) Exhalation without any prevention.

(a.2) Coughing/sneezing without any prevention.

(b.1) Exhalation during the ophthalmic examination.

(b.2) Coughing/sneezing during the ophthalmic examination.

The configurations for these cases are depicted in Fig. 2. In these cases, the upper torso of the manikins was modeled as rigid bodies with three-dimensional 4-node, bilinear quadrilateral R3D4 elements provided in Abaqus/Explicit. The same elements were valid for the protective breath shields. The outer skin of the manikins and protective shield surfaces were modeled by using the rough friction model in conjunction with the no-separation contact pressure-overclosure relationship [14]. Therefore, the particles hitting the surfaces were assumed to stick rather than bouncing back.

As seen in the exhalation case of the first scenario (Fig. 3a), for which the exit flow was $\sim 1000 \mathrm{~mm} / \mathrm{s}$, the average horizontal distance of the flow front was computed as $\sim 200 \mathrm{~mm}$, while the 
a
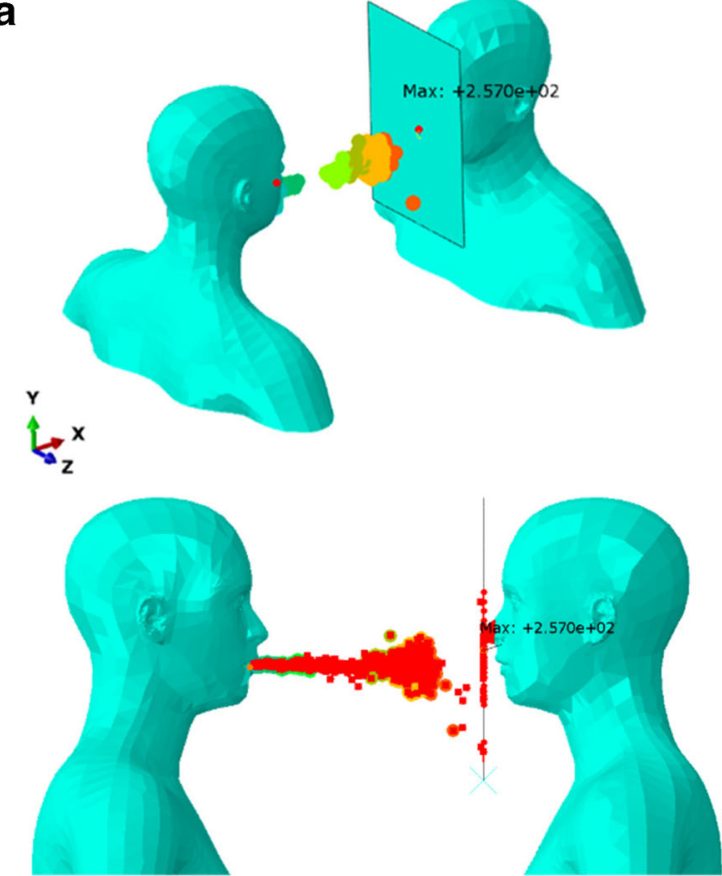

$\stackrel{r}{a} \rightarrow x$

b
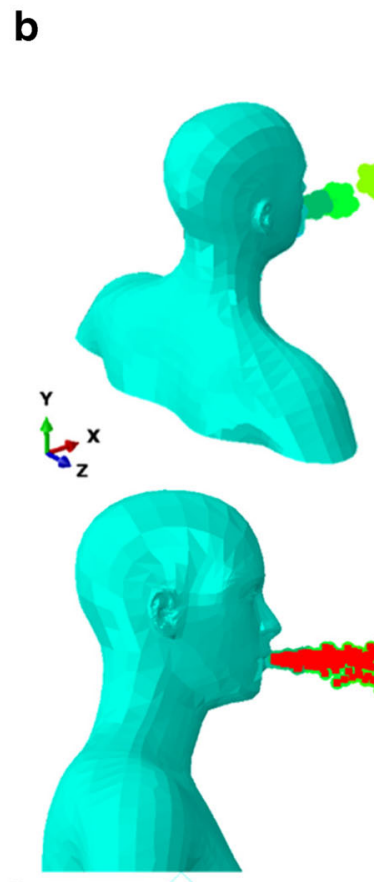

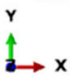

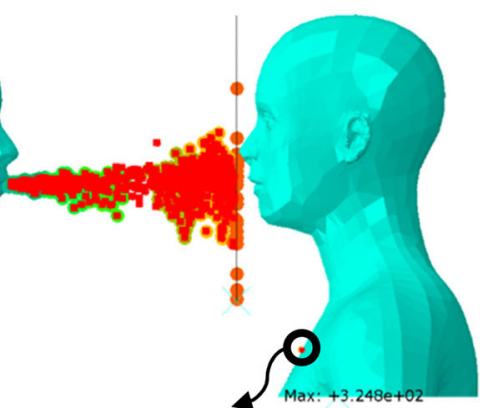

Particles beyond the protective breath shield

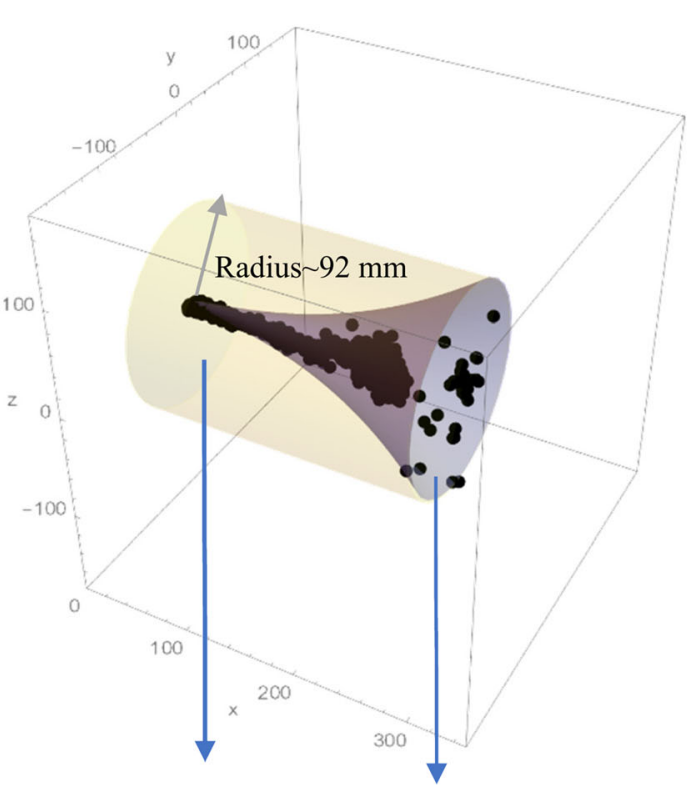

Potential zone Effective zone of risk of risk

Fig. 4 Airborne particle projection during the ophthalmic examination with the protective breath shield: a exhalation (the maximum flow velocity at the exit from the patient's mouth is $\sim 1000 \mathrm{~mm} / \mathrm{s}$ ), b coughing/sneezing (the maximum flow velocity at the exit from the patient's mouth is $\sim 12,000 \mathrm{~mm} / \mathrm{s}$ )

individual particles can reach up to a horizontal distance of $500 \mathrm{~mm}$. Hence, it can be deduced that if the social distancing is not followed, there is a risk of airborne pathogen spreading even for the act of exhalation. The case for coughing or sneezing (Fig. 3b) is unquestionably more hazardous. For instance, in case of an exit flow of $\sim 12,000 \mathrm{~mm} / \mathrm{s}$ without any preventive action, 

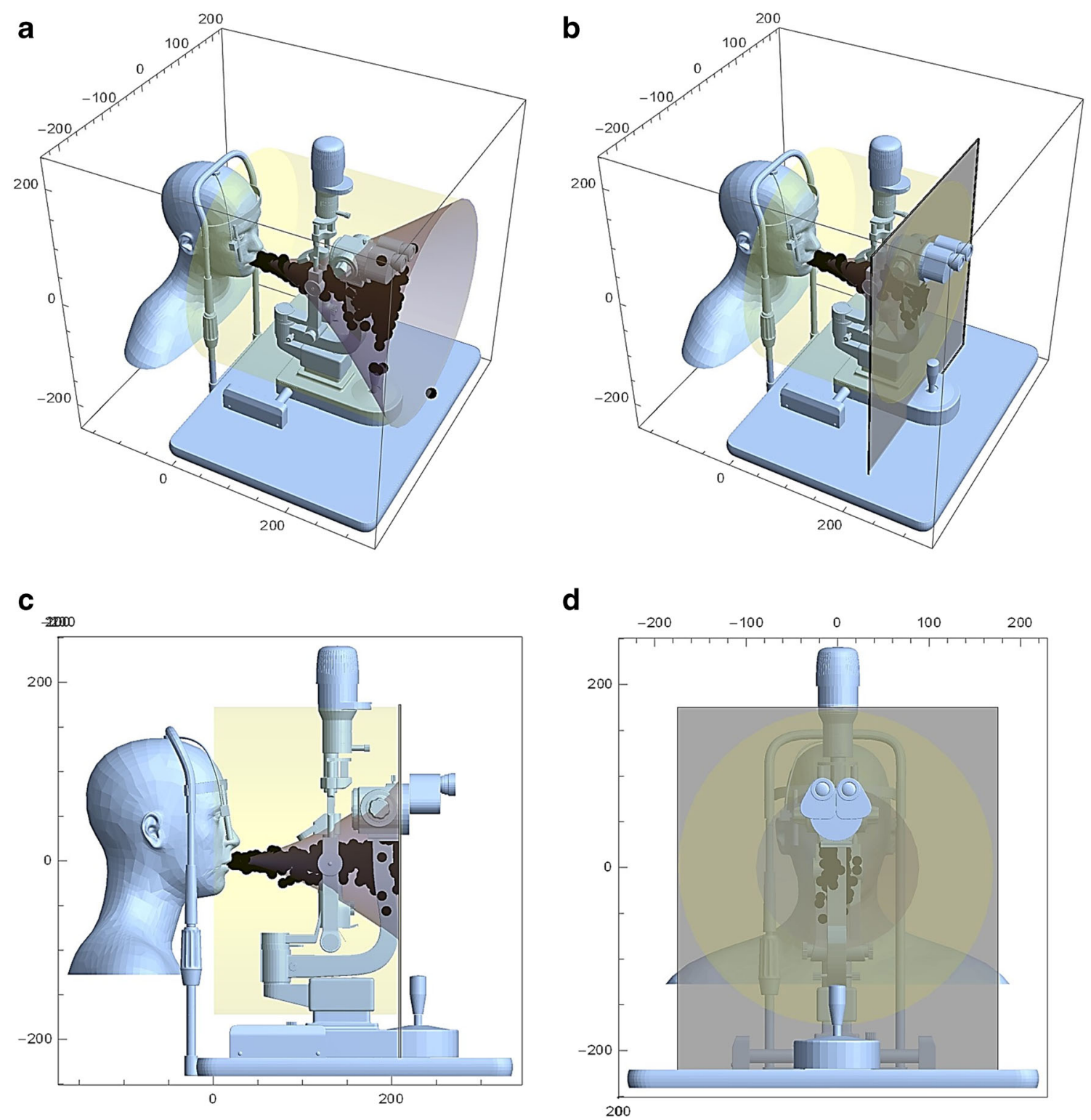

Fig. 5 Conceptual shield design based on the simulation results: a no shield, b effect of the shield in case of coughing/sneezing (isometric view), $\mathbf{c}$ front view of the shield, $\mathbf{d}$ examiner's view

the average horizontal distance of the flow front was obtained to be $\sim 1300 \mathrm{~mm}$. Even more critical than this, some of the particles flew a horizontal distance of nearly $3000 \mathrm{~mm}$ in the time span of $t=1.0 \mathrm{~s}$, which is greater than the social distancing recommendations, with the potent risk of carrying pathogens further than the flow front [4].

The results for the abovementioned cases show the necessity of preventive measures. Especially, in case of medical examinations such as ophthalmic examination, for which the ophthalmologist and the patient have a distance around 200$250 \mathrm{~mm}$. For this reason, protective breath shields that are mounted to the biomicroscopes have been in use (Fig. 1b). These shields approximately have the size of an A4 paper $(210 \mathrm{~mm} \times 297 \mathrm{~mm})$ and are placed very close to the ophthalmologist. However, there is no explicit recommendation/guideline regarding the size for these sheets and very little is known about the projection of airborne pathogens during the close proximity medical examinations even there exists protection. In consideration of this issue, a case study on such examination was carried out, the configuration of which is depicted in Fig. 4.

In the case of exhalation (Fig. 4a, clip 1), it was deduced from the simulations that the protective breath shield configuration works well and most of the particles at the flow front stick to the shield that was assumed to have a rough surface. However, in case of coughing/sneezing (Fig. 4b, clip 2), it was observed that particles may flow beyond the protective shield and may get in contact with the ophthalmologist clothing, 
indicating a tangible risk. In order to minimize the risks, it appears that larger shields or more strict preventive measures are needed.

Based on the simulations, the minimum radius for a new shield design should be $180 \mathrm{~mm}$ as depicted in Fig. 4b. The shield should undoubtedly cover the examiner's head and chest. A conceptual design is proposed based on the minimum size requirements deduced from the simulation data, which can be seen in Fig. 5. The new concept aims at covering the entire risk-zone perimeter and providing protection for the examinee and examiner against viral transmission if no other preventive measures such as wearing masks, goggles, or gowns are taken.

\section{Discussions}

A standard ophthalmologic examination relies strongly on physical evaluation to make a diagnosis. Centers for Disease Control defines close contact for transmission risk as being $2 \mathrm{~m}$ from a patient for examinations that last 1 to $2 \mathrm{~min}$ [35]. The time we spend to complete a detailed ophthalmic examination is far beyond this period. It is therefore important to examine the projection behavior of droplets to determine effective protective measures during our usual practices.

To that end, a three-dimensional model was used in the present study to predict the transmission of droplets during the ophthalmic examination. Normal exhalation, coughing, and sneezing mechanisms with or without protective shields were simulated using different particle injection velocities. Although protective measures like wearing masks, gowns, and goggles are recommended during COVID-19 pandemic, in our study, none of these measures was taken into account for evaluating the sole effect of the breath shield to prevent transmission of airborne pathogens since these measures are difficult to standardize and might be discarded by the patients and also clinicians after the acute influence of the pandemic passes.

The results indicated that the average horizontal distance of the flow even for normal breathing scenarios is enough for viral transmission during the biomicroscopic examination. Although protective breath shields mounted to slit lamps offer a physical barrier between the ophthalmologist and patient, it appears that the dimensions of the currently used shields are inadequate, especially in case of coughing/sneezing. Based on our simulations carried out in the present study, the minimum radius for a shield should be $180 \mathrm{~mm}$, and the shield should cover the examiner's head and chest sections. Having said that, one must admit that approaching the eyelids for a complete view of the ocular structures or fundus examination with a hand-held lens would be challenging with a breath shield of provided dimensions. As a result, a protective shield that allows access for fundoscopy or the ability to approach the eye will not be adequate for the protection of the ophthalmologist on its own and other measures including masks and goggles are necessary in order to perform a routine eye examination. Additionally, thorough periodic cleaning of the protective breath shield and use of disposable isolation gowns, gloves, caps, eye protection, surgical masks, or thermal/chemical disinfection for the reusable laboratory clothing is needed since contaminant particles/droplets can stay in still air for several hours [4].

Numerous measures - such as rapid diagnosis, tracing, and quarantine - have been adopted to counter the SARS-CoV-2 pandemic. However, due to delays in the initial detection of asymptomatic cases and infected individuals in the incubation period, the infection is still exhibiting an uprising trend [28]. As the occurrence and need for awareness of novel epidemic agents have been increasing from year to year, it appears paramount to accumulate knowledge for future outbreaks. Stepping up infection control measures and updating current practices for preventing transmission in an evidence-based process necessitates interdisciplinary work, which requires joint efforts of healthcare professionals, engineers, and civil servants [36]. The present study aimed to serve as a starting platform for research into technical developments to reduce viral transmission during patient examination.

Acknowledgments This work used computational and storage services associated with the Hoffman2 Shared Cluster provided by UCLA Institute for Digital Research and Education's Research Technology Group. In this work, A.K. conducted the numerical analyses, A.K. and B.C.C. conceptualized and wrote the manuscript. E.T. provided technical guidance and editorial supervision.

Availability of data and material (data transparency) All data and software applications support our published claims and comply with field standards.

Authors' contributions All authors have participated sufficiently in the preparation of this work to take public responsibility for it.

\section{Compliance with ethical standards}

Conflicts of interests The authors declare that they have no conflict of interest.

Ethics approval This article does not contain any studies with human participants or animals performed by any of the authors.

Informed consent Consent has been granted by the individuals for use of the Fig. 1a in the submission to the journal.

\section{References}

1. Wang C, Horby PW, Hayden FG, Gao GF (2020) A novel coronavirus outbreak of global health concern. Lancet 395(10223):470 473 
2. Callaway E, Cyranoski D (2020) China coronavirus: six questions scientists are asking. Nature 577(7792):605

3. Organisation WH (2020) Statement on the second meeting of the international health regulations (2005) emergency committee regarding the outbreak of novel coronavirus (2019-nCoV). https:// www.who.int/news-room/detail/30-01-2020-statement-on-thesecond-meeting-of-the-international-health-regulations-(2005)emergency-committee-regarding-the-outbreak-of-novelcoronavirus-(2019-ncov). Accessed 18 April 2020

4. Organisation WH (2020). Infection prevention and control during health care when novel coronavirus ( $\mathrm{nCoV}$ ) infection is suspected. https://www.who.int/publications-detail/infection-prevention-andcontrol-during-health-care-when-novel-coronavirus-(ncov)infection-is-suspected-20200125. Accessed 18 April 2020

5. Holmes KV (2003) SARS-associated coronavirus. N Engl J Med 348(20):1948-1951

6. Otter J, Donskey C, Yezli S, Douthwaite S, Goldenberg S, Weber D (2016) Transmission of SARS and MERS coronaviruses and influenza virus in healthcare settings: the possible role of dry surface contamination. J Hosp Infect 92(3):235-250

7. Wang B, Zhang A, Sun J, Liu H, Hu J, Xu L (2005) Study of SARS transmission via liquid droplets in air. J Biomech Eng 127(1):32-38

8. Chang D, Xu H, Rebaza A, Sharma L, Cruz CSD (2020) Protecting health-care workers from subclinical coronavirus infection. Lancet Respir Med 8(3):13

9. Zhou Y, Zeng Y, Tong Y, Chen C (2020) Ophthalmologic evidence against the interpersonal transmission of 2019 novel coronavirus through conjunctiva. MedRxiv. https://doi.org/10.1101/2020.02. 11.20021956

10. Lu C-w, X-f L, Z-f J (2020) 2019-nCoV transmission through the ocular surface must not be ignored. Lancet 395(10224):39

11. Erdal S, Esment NA (1995) Human head model as an aerosol sampler: calculation of aspiration efficiencies for coarse particles using an idealized human head model facing the wind. J Aerosol Sci 26(2):253-272

12. Stahlhofen W, Rudolf G, James A (1989) Intercomparison of experimental regional aerosol deposition data. J Aerosol Med 2(3): 285-308

13. Monaghan JJ (1992) Smoothed particle hydrodynamics. Annu Rev Astron Astrophys 30(1):543-574

14. Systèmes D (2009) ABAQUS user's manual. Abaqus Standard Version:6.13-11

15. Müller M, Charypar D, Gross M (2003) Particle-based fluid simulation for interactive applications. In: Proceedings of the 2003 ACM SIGGRAPH/Eurographics symposium on Computer animation, Eurographics Association, pp 154-159

16. Trimulyono A, Hashimoto H (2019) Experimental validation of smoothed particle hydrodynamics on generation and propagation of water waves. J Mar Sci Eng 7(1):17

17. Wan H, Li R, Pu X, Zhang H, Feng J (2017) Numerical simulation for the air entrainment of aerated flow with an improved multiphase SPH model. Int Comput Fluid Dyn 31(10):435-449

18. Wieth L, Kelemen K, Braun S, Koch R, Bauer H-J, Schuchmann HP (2016) Smoothed particle hydrodynamics (SPH) simulation of a high-pressure homogenization process. Microfluid Nanofluid 20(2):42

19. Paiva A, Petronetto F, Lewiner T, Tavares G (2006) Particle-based non-Newtonian fluid animation for melting objects. In: 19th Brazilian Symposium on Computer Graphics and Image Processing, IEEE, pp 78-85

20. Kelager M (2006) Lagrangian fluid dynamics using smoothed particle hydrodynamics. University of Copenhagen: Department of Computer Science 2
21. Monaghan J (1989) On the problem of penetration in particle methods. J Comput Phys 82(1):1-15

22. Ahmadzadeh M, Saranjam B, Fard AH, Binesh AR (2014) Numerical simulation of sphere water entry problem using Eulerian-Lagrangian method. Appl Math Model 38(5-6):16731684

23. Bunyan D, Ritchie L, Jenkins D, Coia J (2013) Respiratory and facial protection: a critical review of recent literature. J Hosp Infect 85(3):165-169

24. Fernstrom A, Goldblatt M (2013) Aerobiology and its role in the transmission of infectious diseases. J Pathog 2013:493960

25. Loudon RG, Roberts RM (1967) Droplet expulsion from the respiratory tract. Am Rev Respir Dis 95(3):435-442

26. Papineni RS, Rosenthal FS (1997) The size distribution of droplets in the exhaled breath of healthy human subjects. J Aerosol Med 10(2):105-116

27. Li J-PO, Lam DSC, Chen Y, Ting DSW (2020) Novel coronavirus disease 2019 (COVID-19): the importance of recognising possible early ocular manifestation and using protective eyewear. Br J Ophthalmol 104(3):297-298

28. Herfst S, Böhringer M, Karo B, Lawrence P, Lewis NS, Mina MJ, Russell CJ, Steel J, de Swart RL, Menge C (2017) Drivers of airborne human-to-human pathogen transmission. Curr Opin Virol 22: 22-29

29. Tang JW, Nicolle A, Pantelic J, Koh GC, De Wang L, Amin M, Klettner CA, Cheong DK, Sekhar C, Tham KW (2012) Airflow dynamics of coughing in healthy human volunteers by shadowgraph imaging: an aid to aerosol infection control. PLoS One 7(4):34818

30. Wei J, Li Y (2017) Human cough as a two-stage jet and its role in particle transport. PLoS One 12(1):0169235

31. Malvè M, Del Palomar AP, López-Villalobos J, Ginel A, Doblaré M (2010) FSI analysis of the coughing mechanism in a human trachea. Ann Biomed Eng 38(4):1556-1565

32. Geoghegan P, Laffra A, Hoogendorp N, Taylor M, Jermy M (2017) Experimental measurement of breath exit velocity and expirated bloodstain patterns produced under different exhalation mechanisms. Int J Legal Med 131(5):1193-1201

33. Nascimento WV, Cassiani RA, Dantas RO (2012) Gender effect on oral volume capacity. Dysphagia 27(3):384-389

34. Zhang B, Zhu C, Ji Z, Lin C-H (2017) Design and characterization of a cough simulator. J Breath Res 11(1):016014

35. Centers for Disease Control and Prevention (2020). Interim US guidance for risk assessment and public health management of persons with potential Coronavirus Disease 2019 (COVID-19) exposures: geographic risk and contacts of laboratory-confirmed cases. https://www.cdc.gov/coronavirus/2019-ncov/php/risk-assessment. html. Accessed 20 March 2020

36. Coker R, Rushton J, Mounier-Jack S et al (2011) Towards a conceptual framework to support one-health research for policy on emerging zoonoses. Lancet Infect Dis 11(4):326-331

This article contains video clips as additional online-only material. The following should appear online-only: Clip 1, Clip 2.

Publisher's note Springer Nature remains neutral with regard to jurisdictional claims in published maps and institutional affiliations. 